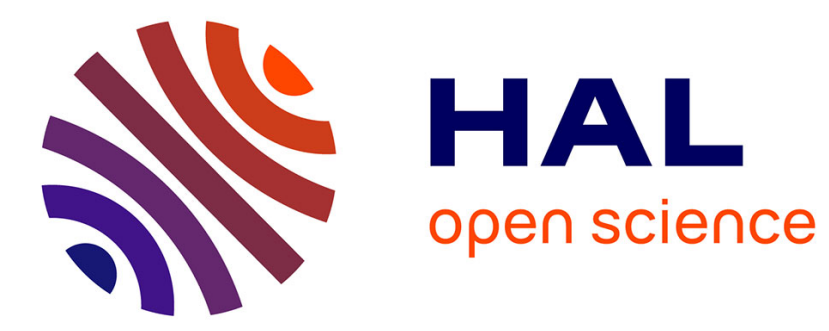

\title{
Probabilistic multiscale models and measurements of self-heating under multiaxial high cycle fatigue
}

Martin Poncelet, Cédric Doudard, Sylvain Calloch, Bastien Weber, François

Hild

\section{- To cite this version:}

Martin Poncelet, Cédric Doudard, Sylvain Calloch, Bastien Weber, François Hild. Probabilistic multiscale models and measurements of self-heating under multiaxial high cycle fatigue. Journal of the Mechanics and Physics of Solids, 2010, 58, pp.578-593. hal-00453966

\section{HAL Id: hal-00453966 https://hal.science/hal-00453966}

Submitted on 6 Feb 2010

HAL is a multi-disciplinary open access archive for the deposit and dissemination of scientific research documents, whether they are published or not. The documents may come from teaching and research institutions in France or abroad, or from public or private research centers.
L'archive ouverte pluridisciplinaire HAL, est destinée au dépôt et à la diffusion de documents scientifiques de niveau recherche, publiés ou non, émanant des établissements d'enseignement et de recherche français ou étrangers, des laboratoires publics ou privés. 


\title{
Probabilistic multiscale models and measurements of self-heating under multiaxial high cycle fatigue
}

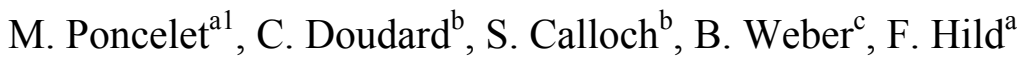 \\ ${ }^{a}$ LMT-Cachan (ENS Cachan/CNRS/Université Paris 6/PRES UniverSud Paris) \\ 61 avenue du Président Wilson, F-94235 Cachan Cedex, France \\ hild@lmt.ens-cachan.fr \\ ${ }^{\text {b}}$ LBMS EA 4325 (E.N.S.I.E.T.A./Université de Brest/E.N.I.B.) \\ 2 rue François Verny, F-29806 Brest Cedex, France \\ cedric.doudard@ensieta.fr, sylvain.calloch@ensieta.fr \\ ${ }^{\mathrm{c}}$ ArcelorMittal Maizières Research \\ Voie Romaine BP 30320, F-57283 Maizières-lès-Metz, France \\ bastien.weber@arcelormittal.com
}

\begin{abstract}
Different approaches have been proposed to link high cycle fatigue properties to thermal measurements under cyclic loadings, usually referred to as "self-heating tests." This paper focuses on two models whose parameters are tuned by resorting to self-heating tests and then used to predict high cycle fatigue properties. The first model is based upon a yield surface approach to account for stress multiaxiality at a microscopic scale, whereas the second one relies on a probabilistic modelling of microplasticity at the scale of slip-planes.

Both model identifications are cost effective, relying mainly on quickly-obtained temperature data in self-heating tests. They both describe the influence of the stress heterogeneity, the volume effect and the hydrostatic stress on fatigue limits. The thermal effects and mean fatigue limit predictions are in good agreement with experimental results for in and out-of phase tension-torsion loadings. In the case of fatigue under non-proportional loading paths, the mean fatigue limit prediction error of the critical shear stress approach is three times less than with the yield surface approach.
\end{abstract}

\section{Keywords}

(A) thermomechanical process, (B) Metallic materials, (C) Mechanical testing , (C) Probability and statistics, Multiaxial Fatigue.

\footnotetext{
${ }^{1}$ Corresponding author.

Present address : CEA Saclay, DEN, SRMA

91191 Gif-sur-Yvette cedex, France

Email: martin.poncelet@cea.fr - Fax: +33 (0)1 69087167.
} 


\section{Nomenclature}

Variables with very close meanings are used in both models. For the sake of clarity, they are written without tilda for the yield surface approach and with tilda $(\sim)$ for the critical shear stress approach. The superscript * refers to any of the two models, e.g. $\Sigma_{\text {effdiss }}{ }^{*}$ corresponds to $\Sigma_{\text {effdiss }}$ for the yield surface approach and $\widetilde{\Sigma}_{\text {effdiss }}$ for the critical shear stress approach, respectively.

\section{$\underline{\text { Stresses }}$}

$\begin{array}{ll}\underline{\underline{\Sigma}} & \text { Macroscopic stress tensor } \\ \underline{\underline{\underline{\Sigma}}} m & \text { Mean macroscopic stress over a cycle } \\ \underline{\underline{\sigma}} & \text { Microscopic stress tensor } \\ \underline{\underline{S}} & \text { Deviatoric stress tensor } \\ \underline{\underline{X}} & \text { Back stress } \\ \underline{I} & \text { Unit second order tensor } \\ J_{2} & \text { Second stress invariant } \\ T & \text { Macroscopic shear stress } \\ T_{0} & \text { Macroscopic shear stress amplitude } \\ I_{1, \max } & \text { Max. hydrostatic stress over a cycle } \\ I_{1, m} & \text { Mean hydrostatic stress over a cycle }\end{array}$

\section{Material parameters}

$\mu \quad$ Shear modulus

$v \quad$ Poisson's ratio

$\rho \quad$ Mass density

c Specific heat

C Hardening parameter

\section{Microplasticity description}

$\lambda, \tilde{\lambda}$ Intensity of the Poisson Point Process $V_{s}, \widetilde{V}_{s}$ Volume around each site

$\underline{\underline{\varepsilon}}^{p} \quad$ Plastic strain tensor

$\dot{\chi} \quad$ Plastic multiplier

$h, \tilde{h} \quad$ Microscopic hardening coefficients

$\sigma_{y} \quad$ Yield stress

$\tau \quad$ Shear stress

$\tau_{y} \quad$ Critical shear stress

a Slip direction

$n \quad$ Normal to the considered plane

$m \quad$ Slip direction in the plane

$\gamma^{p} \quad$ Plastic slip

$\kappa(m) \quad$ Activated directions distribution factor

\section{Heat transfer parameters and variables}

$\delta, \widetilde{\delta} \quad$ Intrinsic dissipated energies of one site over a cycle

$\Delta \quad$ Global (mean) dissipated energy

$D \quad$ Total dissipated energy

$\tau_{e q} \quad$ Characteristic time

$\theta \quad$ Mean temperature variation

$\bar{\theta} \quad$ Mean steady-state temperature

\section{Fatigue parameters and variables}

$P_{F} \quad$ Failure probability

$V_{\text {eff }} \quad$ Effective volume

$\bar{\Sigma}_{\infty}, \widetilde{\bar{\Sigma}}_{\infty}$ Mean fatigue stress

$\overline{\bar{\Sigma}}_{\infty} \quad$ Standard deviation of the fatigue stress

$C V \quad$ Coefficient of variation

Model parameters to be identified

$m, \widetilde{m} \quad$ Weibull modulus

$\eta, \tilde{\eta} \quad$ Thermal scale parameter

$\alpha, \widetilde{\alpha} \quad$ Hydrostatic stress effect parameter

$\widetilde{V}_{0}\left(\widetilde{S}_{0}\right)^{m}, \widetilde{V}_{0}\left(\widetilde{S}_{0}\right)^{m} \quad$ Fatigue scale parameter

\section{Heterogeneity factors and equivalent stress}

$\Sigma_{0}^{e q}, \widetilde{\Sigma}_{0}^{e q} \quad$ Equivalent stress amplitude

$\Sigma_{\text {effdiss }}, \widetilde{\Sigma}_{\text {effdiss }}$ Effective dissipative stress

$G_{m+2}, \widetilde{G}_{m+2} \quad$ Dissipation heterogeneity factor

$H_{m+2}, \widetilde{H}_{m+2} \quad$ Stress heterogeneity factor

\section{Loading description}

$\Sigma_{11,0} \quad$ Tensile stress amplitude

$\Sigma_{12,0} \quad$ Shear stress amplitude

$\phi \quad$ Ratio between shear and normal stresses

$r \quad$ Radius of the specimen

Re External radius of the specimen

$f_{r} \quad$ Frequency of loading

$\varphi \quad$ Phase lag between shear and normal stresses 


\section{1-Introduction}

The constant improvement of materials and the industrial need for appropriate characterisation lead to countless tests. This fact is all the more penalizing in the case of High Cycle Fatigue (HCF) where experiments last more than a week and use several tens of specimens to get a Wöhler diagram with different reliability levels. This problem is even more important when multiaxial fatigue is concerned, insofar as each loading path corresponds to a specific Wöhler campaign. Consequently, alternative and faster methods of fatigue characterisation are sought to save time.

A promising alternative is based on the measurement of the specimen temperature changes during cyclic loadings (Bérard et al., 1998; Cazaud, 1948; Cura et al., 2005; Dengel and Harig, 1980; Doudard et al., 2004; Galtier et al., 2002 ; Harry et al., 1981; Kanarchuk et al., 1989; Krapez et al., 1999; La Rosa and Risitano, 2000; Lehr, 1926; Luong, 1995; Mabru and Chrysochoos, 2001; Moore and Kommers, 1921; Stärk, 1980; Stromeyer, 1915; Welter, 1937; Yang et al., 2005). Various experimental procedures were proposed involving the use of thermocouples (Doudard et al., 2004; Galtier et al., 2002; Lehr, 1926; Moore and Kommers, 1921; Welter, 1937) or thermography (Bérard et al., 1998; Cura et al., 2005; Krapez et al., 1999; La Rosa and Risitano, 2000; Luong, 1995; Mabru and Chrysochoos, 2001; Yang et al., 2005), and focusing on the transient temperature history (Krapez et al., 1999; Moore and Kommers, 1921) and / or the steady-state temperature reached after several thousands of cycles (Doudard et al., 2004; Galtier et al., 2002; La Rosa and Risitano, 2000; Luong, 1995; Mabru and Chrysochoos, 2001). The particular test used herein, referred to as "self-heating test," consists in applying successive series of cycles (around 3000 cycles for each amplitude step) for different increasing stress amplitudes to a single specimen. For some materials (e.g., steels), the fact that the stress level leading to a significant increase of the steady-state temperature is correlated to the mean fatigue limit has been exploited early on (Lehr, 1926; Welter, 1937). More recently Doudard et al. (2005) have proposed a two-scale model (i.e., elastoplastic inclusions embedded in an elastic matrix) to explain this relationship. It is assumed that fatigue initiation is caused by microplasticity that induces dissipated energy. This type of view point was proposed by Dang Van (1973). The originality of the present approach lies in the identification method, namely, all the material parameters introduced in the model are tuned using the self-heating test under cyclic loading (Doudard et al., 2005). 
Moreover fatigue results are scattered and this phenomenon is not taken into account by classical two-scale (i.e., deterministic) approaches. To describe the scatter, a probabilistic two-scale model (i.e., a set of elastoplastic sites randomly distributed within an elastic matrix) has been proposed. The distribution of sites is determined from self-heating measurements under cyclic loadings (Doudard et al., 2004). This identification procedure was validated for several materials (Doudard, 2004; Poncelet, 2007) by comparing the prediction of S/N curves given by the model and experimental results for uniaxial stress states.

The extension of this self-heating based approach to multiaxial loadings (i.e., analysis of the self-heating test to identify a multiaxial fatigue criterion) is not easy and presents two challenges, namely, performing and understanding self-heating tests under multiaxial cyclic loadings, and second, the definition of the activation (equivalent) stress of elastoplastic sites introduced in the probabilistic two-scale model. A first response was obtained from $a$ heat transfer point of view under proportional cyclic loadings (Doudard et al., 2007a) and nonproportional cyclic loadings (Poncelet et al. 2007) whereby a unique thermal response for different loading types is sought in the stress space. Concerning the microplasticity description in a two-scale model, two different approaches are generally used, namely, by following a "yield surface approach" (e.g., von Mises' yield criterion (Doudard et al., 2007a; Lemaitre and Doghri, 1994)) or a "critical shear stress approach" (e.g., using Schmid's law (Dang Van, 1973; Doudard et al., 2007b; Morel, 1998)). With a deterministic point of view (i.e., the microplastic activation is deterministic), no real difference is obtained between both descriptions in terms of (good) predictions of fatigue properties under proportional loadings. However, unsatisfactory mean fatigue limit predictions are observed under non-proportional loading histories (Bainvillet et al., 2003). With a probabilistic point of view (i.e., the microplastic activation is a random process), and to the best of the authors' knowledge, no conclusion was drawn. Thus, this work has two main goals. The first one is to demonstrate the potential of a probabilistic scenario in the description of the microplastic activity to improve the prediction of multiaxial fatigue properties of materials. The second goal of this work is to propose an identification procedure of the material parameters based on selfheating measurements under cyclic loadings.

The present paper is divided into two parts. The first part is dedicated to the description of the two models based on a probabilistic description of microplastic activation. Yield surface and critical shear stress approaches descriptions are introduced, i.e., from the assumptions concerning microplasticity activation to the thermal and fatigue responses. Theses two models are limited to the prediction of crack initiation as the previous ones 
proposed by the authors. In the second part, an application to tension-torsion cyclic loadings is described. The identification procedure is performed using only two self-heating test results obtained for different loading paths, and one uniaxial mean fatigue limit. Both approaches give good thermal predictions for other loading paths. The predictions of multiaxial fatigue properties with both models are analysed and then compared with experimental results.

\section{2-Two probabilistic multiscale models}

The main goal of this section is to introduce two models based on probabilistic approaches. The physical process of damage initiation is here assumed to be governed by intragranular microplasticity. At that scale, local fields experience fluctuations due to the polycrystalline nature of the materials considered. When a local equivalent stress (to be specified) exceeds a local threshold, microplastic activity starts. The joint effects of local stress fluctuations and of local weaknesses are accounted for through a stress-dependent activation probability. It is worth noting that if another mechanism (e.g., microcrack initiation and propagation), or another scale at which the degradation occurs (e.g., grain clusters), the equivalent stress and activation probability may change.

A set of elastoplastic sites randomly distributed within an elastic matrix is considered. In the present case, no spatial correlations are considered, even though more elaborate hypotheses can be made (Jeulin, 1991; Sobczyk and Kirkner, 2001). It is assumed that HCF damage is localized at the mesoscopic scale and is induced by microplastic activity (in the grains whose orientation is favourable). The distribution of active sites (i.e., sites where microplasticity occurs) of volume $V_{s}$ in relation to their surrounding explains the 'probabilistic' feature, which is modelled by a Poisson Point Process (Curtin, 1991; Gulino and Phoenix, 1991; Jeulin, 1991; Fedelich, 1998; Denoual and Hild, 2002). The probability of finding $k$ active sites in a domain $\Omega$ of volume $V$ reads

$$
P_{k}^{*}(\Omega)=\frac{\left[-\lambda^{*} V\right]^{k}}{k !} \exp \left[-\lambda^{*} V\right],
$$

where $\lambda^{*}$ is the intensity of the Poisson Point Process and $\lambda^{*} V$ is the mean number of active sites. The form of the intensity of the process is linked to the modelling of microplasticity, its value depends on the loading level, and will be described in Section 2.1. 
The relationship between the stress tensor in a site where microplasticity occurs, $\underline{\underline{\sigma}}$, and the macroscopic stress tensor $\underline{\underline{\Sigma}}$ is given by the localisation law (Berveiller and Zaoui, 1979; Kröner, 1984)

$$
\underline{\underline{\sigma}}=\underline{\underline{\Sigma}}-2 \mu(1-\beta) \underline{\underline{\varepsilon}}^{p}
$$

where $\underline{\varepsilon}^{p}$ is the corresponding plastic strain tensor (an additive decomposition of strain with an elastic and a plastic part is assumed for each site) and $\mu$ the shear modulus. $\beta=\frac{2(4-5 v)}{15(1-v)}$ is given by Eshelby's analysis of a spherical inclusion in an elastic matrix, where $v$ denotes the corresponding Poisson's ratio (Eshelby, 1957).

In the next section, two different approaches describing microplasticity (i.e., the elastoplastic behaviour of sites) are presented. The first one uses a yield surface (e.g., based upon von Mises' equivalent stress) and the second one a critical shear stress approach (e.g., Schmid's criterion).

\section{1-Elastoplastic behaviour of the sites}

\subsection{1- Yield surface approach}

The first model presented herein is an extension of the one initially proposed in Refs. (Doudard et al., 2005, 2007a ; Poncelet et al., 2007). The uniaxial model (Doudard et al., 2004) needs a reduced number of parameters and allows closed-form solutions to be derived for the description of self-heating responses and the number of cycles to failure in HCF. The present model uses the same type of assumptions so that most of these advantages remain.

Microplasticity is modelled at a microscopic scale and is described by a yield surface

$$
f=J_{2}(\underline{\underline{S}}-\underline{\underline{X}})-\sigma_{y} \leq 0
$$

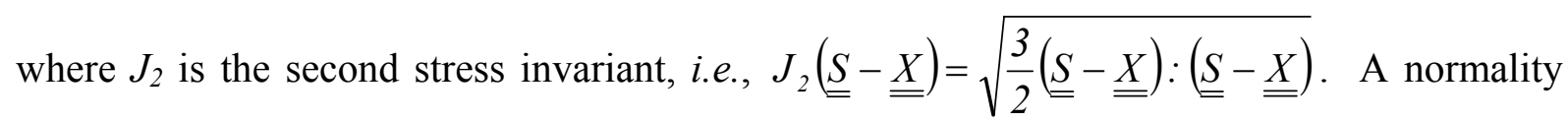
rule is assumed and linear kinematic hardening is considered

$$
\begin{gathered}
\stackrel{\dot{\varepsilon}}{=}^{p}=\dot{\chi} \frac{\partial f}{\partial \underline{\underline{S}}}, \\
\underline{\underline{X}}=\frac{2}{3} C \underline{\underline{\dot{\varepsilon}}}^{p} \quad \text { and } \quad \underline{\underline{X}}\left(\underline{\varepsilon}^{p}=\underline{\underline{0}}\right)=\underline{\underline{0}} .
\end{gathered}
$$


The magnitude of the intrinsic dissipated energy $\delta\left(\Sigma_{0}^{\mathrm{eq}}, \sigma_{\mathrm{y}}\right)$ in a site over a loading cycle is calculated for a given value of the yield stress $\sigma_{y}$, von Mises' equivalent stress amplitude $\Sigma_{0}^{e q}=\operatorname{Max}_{t} J_{2}(\underline{\underline{\Sigma}}(t)-\underline{\underline{\Sigma}})$, and a mean stress $\underline{\underline{\Sigma_{m}}}$ given by

$$
\underline{\underline{\Sigma}}_{m}=\underline{\underline{S}}_{m}+I_{1, m} \underline{\underline{I}}=\operatorname{Min}_{Y}\left[\operatorname{Max}_{t} J_{2}(\underline{\underline{\Sigma}}(t)-\underline{\underline{Y}})\right]+\frac{1}{3} \operatorname{Min}_{z}[\operatorname{Max}(\operatorname{trace}(\underline{\underline{\underline{(}}}(t))-z)] \underline{\underline{\underline{I}}} .
$$

For a proportional loading condition, the intrinsic dissipated energy of one site reads

$$
\delta\left(\Sigma_{0}^{e q}, \sigma_{y}\right)=\frac{4 V_{S} \sigma_{y}}{h}\left\langle\Sigma_{0}^{e q}-\sigma_{y}\right\rangle
$$

where $\langle$.$\rangle are Macauley's brackets (i.e., positive part of '.'), h=C+3 \mu(1-\beta)[19]$.

Since $\sigma_{y}$ is assumed to be a random variable, it is proposed that the intensity of the Poisson Point Process describing the activation of microplasticity follows a power law of the equivalent stress amplitude

$$
\lambda=\frac{1}{\mathrm{~V}_{0}}\left(\frac{\Sigma_{0}^{e q}}{S_{0}+\alpha I_{1, m}}\right)^{m},
$$

where $\alpha, m$ and $V_{0} S_{0}{ }^{m}$ are three material parameters, and $I_{1, m}$ the mean hydrostatic stress over a given cycle. Von Mises' equivalent stress amplitude is chosen because of the isotropy of the material tested hereafter. In the case of anisotropy, a more adequate equivalent stress amplitude may be considered. The power-law dependence is chosen for both experimental and theoretical reasons. It was shown that the onset of microplasticity follows a power-law of the applied stress (Cugy and Galtier, 2002), and introducing a power-law as the intensity of a Poisson Point Process leads to a Weibull model when the weakest link assumption is made (Doudard et al., 2004, 2005). The hydrostatic stress dependence is introduced to account for the mean stress effect on self-heating measurements and on fatigue properties. Figure 1 shows schematically the activation scenario of sites, namely, the higher the stress level, the larger the number of activated sites, and therefore the higher the dissipated energy. 


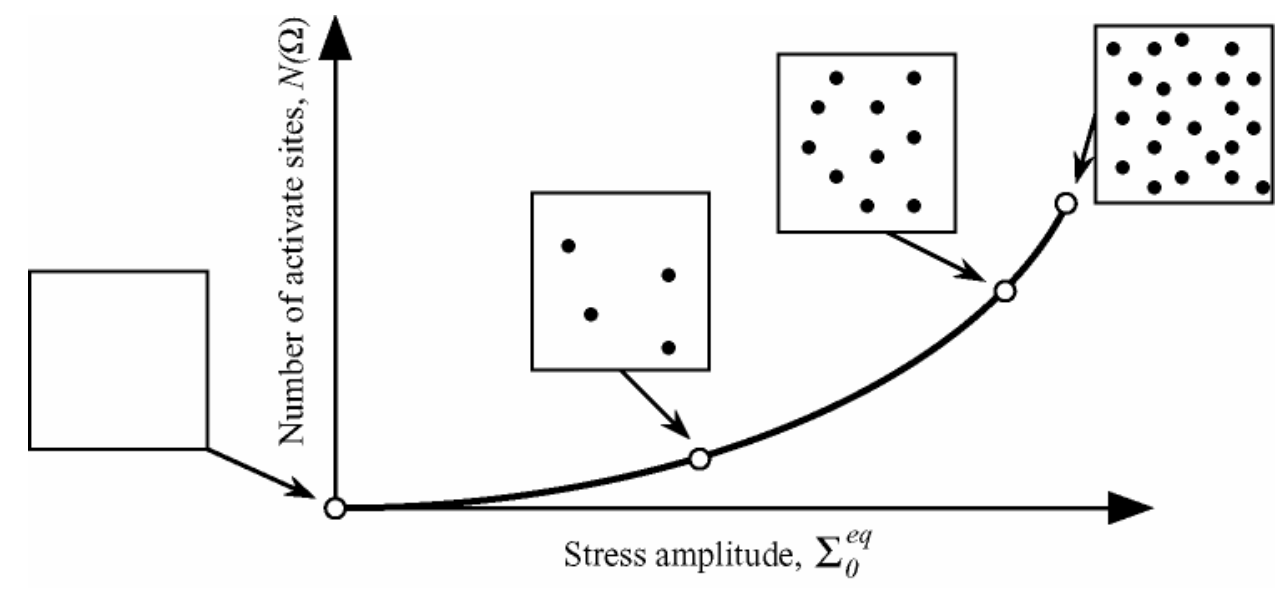

Figure 1: Schematic view of the activation scenario of elastoplastic sites with the equivalent stress amplitude.

The total dissipated energy $D\left(\sum_{0}^{e q}\right)$ is then obtained by integration over the whole population of active sites (Doudard et al., 2005). For a Poisson point process, $\frac{d \lambda}{d \Sigma} V d \Sigma$ corresponds to the mean number of sites activated for a stress amplitude ranging from $\Sigma$ and $(\Sigma+\mathrm{d} \Sigma)$ in an examination domain of volume $V$, i.e. the number of sites whose yield stress lies between $\Sigma$ and $(\Sigma+\mathrm{d} \Sigma)$, and whose dissipated energy during a loading cycle is $\delta\left(\Sigma_{0}^{e q}, \sigma_{y}\right)$. Consequently $D\left(\Sigma_{0}^{e q}\right)$ is expressed as

$$
D\left(\Sigma_{0}^{e q}\right)=\int_{0}^{\Sigma_{0}^{e q}} \delta\left(\Sigma_{0}^{e q}, \Sigma\right) \frac{d \lambda\left(\Sigma_{0}^{e q}\right)}{d \Sigma} V d \Sigma .
$$

For a heterogeneous stress field over a domain $\Omega$ of volume $V$, the general expression of the global (mean) dissipated energy $\Delta$ reads

$$
\Delta=\frac{1}{V} \int_{\Omega} D\left(\Sigma_{0}^{e q}(M)\right) d V .
$$

The corresponding expression in the particular case of a uniform tensile / compressive loading $\left(R=\frac{\Sigma_{\min }}{\Sigma_{\max }}=-1\right)$ for a given value of the stress amplitude $\Sigma_{0}$ reads

$$
\Delta_{\text {unif }}^{\text {tensile }}=\frac{4 m V_{S}}{h(m+1)(m+2)} \frac{\left(\Sigma_{0}\right)^{m+2}}{V_{0} S_{0}^{m}} .
$$

For proportional loadings, one introduces a dissipation heterogeneity factor $G_{m+2}$ defined by 


$$
G_{m+2}=\frac{1}{\mathrm{~V}} \int_{\Omega}\left(\frac{S_{0}+\alpha I_{1, m M}}{S_{0}+\alpha I_{1, m}(M)}\right)^{m}\left(\frac{\Sigma_{0}^{e q}(M)}{\Sigma_{0 M}^{e q}}\right)^{\mathrm{m}+2} \mathrm{dV}
$$

where $\Sigma_{0 M}^{e q}=\max _{M \in \Omega}\left[\Sigma_{0}^{e q}(M)\right]$ and $I_{1, m M}=\max _{M \in \Omega}\left[I_{1, m}(M)\right\rfloor$ to get the same type of expression given in Equation (11), yet valid for any considered history. This factor is equal to 1 in the case of uniform loadings and becomes less than 1 in the case of heterogeneous loadings (e.g., torsion or rotary bending).

It is then possible to express $\Delta^{\text {prop }}$ as

$$
\Delta^{\text {prop }}=\frac{4 m V_{S}}{h(m+1)(m+2)} \frac{\left(\Sigma_{\text {effiss }}\right)^{m+2}}{V_{0} S_{0}^{m}}
$$

where

$$
\Sigma_{\text {effiss }}=\frac{G_{m+2}^{1 /(m+2)} \Sigma_{0 M}^{e q}}{\left(1+\frac{\alpha}{S_{0}} I_{1, m M}\right)^{m /(m+2)}},
$$

is the "effective dissipation stress." For non proportional loadings, it is no longer possible to simplify $\Delta$ because of the expression of $D\left(\Sigma_{0}^{e q}(M)\right) . \Delta$ is then calculated with the same hypothesis, but using a numerical integration scheme. However, it is possible to obtain the same expressions (13) and (14) by introducing the dissipation heterogeneity factor given by

$$
G_{m+2}=\frac{\Delta}{\Delta_{M}}
$$

where $\Delta_{M}$ denotes the mean dissipated energy for a proportional and uniform loading condition, a von Mises' equivalent stress amplitude $\Sigma_{0 M}^{e q}$, and a mean hydrostatic stress $I_{1, m M}$. The use of $\Sigma_{\text {effiss }}$ is helpful since the form of $\Delta$ in Equation (14) is the same for every loading path. In other words, $\Sigma_{\text {effiiss }}$ takes into account the different features of the loading, be it uniaxial or multiaxial, with or without mean stress, uniform or heterogeneous, proportional or non-proportional.

\subsection{2- Critical shear stress approach}

The only -and important- difference with the previous model is the description of microplasticity. Microplasticity is now modelled at the scale of slip planes on which both shear and hydrostatic stresses are taken into account (Doudard et al., 2007b). For the sake of 
clarity, the $\sim$ notation is exclusively used for every variable of the present model having an equivalent in the yield surface approach (e.g., $\Sigma_{0}^{e q}$ and $\left.\widetilde{\Sigma}_{0}^{e q}\right)$. Identical parameters (e.g., Lamé's coefficient $\mu$ ) are unchanged.

Microplasticity is modelled at the scale of slip planes based on Schmid's criterion

$$
\tau-\tau_{y} \leq 0
$$

with $\tau=\underline{\underline{\sigma}}: \underline{\underline{a}}$ the (resolved) shear stress for the considered direction defined by

$$
\underline{\underline{a}}=\frac{1}{2}\left(\underline{n}^{t} \underline{m}+\underline{m}^{t} \underline{n}\right)
$$

where $\underline{n}$ is the direction normal to the considered plane, and $\underline{m}$ the in-plane slip direction. The shear stress $\tau$ for the considered direction is related to the macroscopic shear stress $T$ by the same localization law as before (Doudard et al., 2007b)

$$
\tau=T-\mu(1-\beta) \gamma^{p}
$$

where $\gamma^{p}$ is the plastic slip $\left(\underline{\underline{\varepsilon}}^{p}=\gamma^{p} \underline{\underline{a}}\right)$.

The intrinsic dissipated energy in one site of volume $\widetilde{V}_{S}$ during a cycle for a given critical (resolved) shear stress $\tau_{y}$ and shear amplitude $T_{0}$ (Figure 2) for the considered site direction is expressed as (Doudard et al., 2007b)

$$
\widetilde{\delta}\left(T_{0}, \tau_{y}\right)=\frac{4 \widetilde{V}_{S}}{\widetilde{h}} \tau_{y}\left\langle T_{0}-\tau_{y}\right\rangle,
$$

with $\tilde{h}=\mu(1-\beta)$.

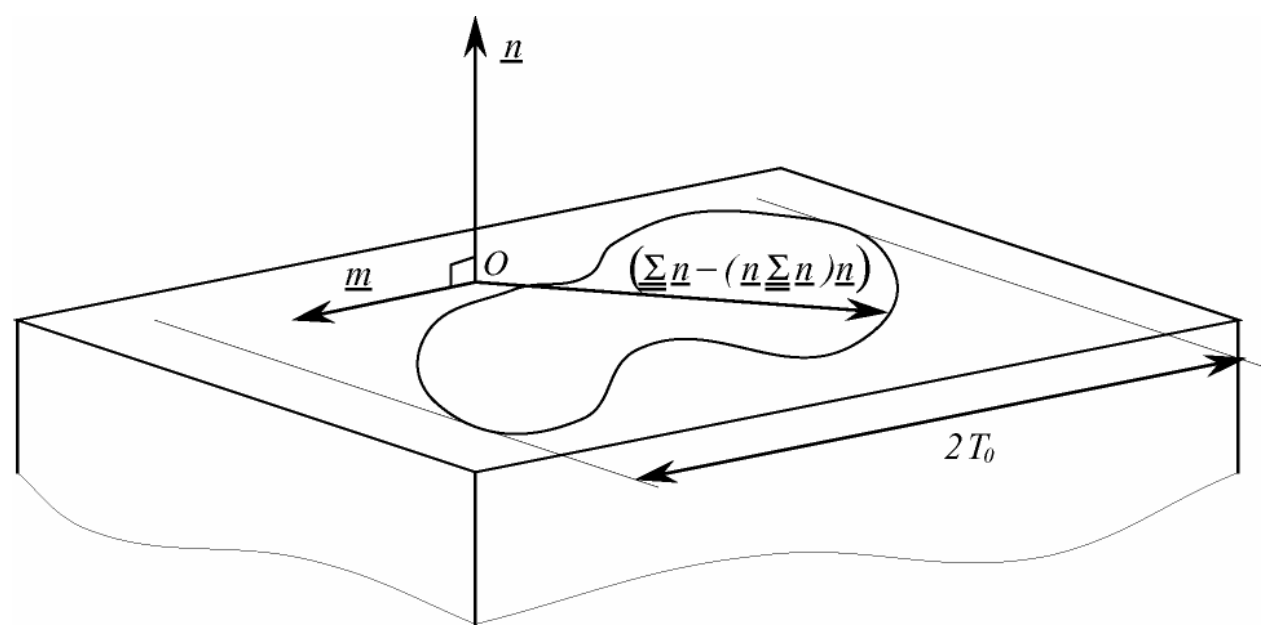

Figure 2: Shear amplitude $T_{0}$ in the plane of normal $\underline{n}$ and slip direction $\underline{m}$. 
Consequently one direction becomes active when the shear stress amplitude $T_{0}$ is greater than the critical shear stress $\tau_{y}$, which is assumed to be a random variable. The intensity of the Poisson Point Process now follows a power law of the macroscopic shear amplitude integrated over all directions in space (defined by the solid angle $\Theta$ ) (Doudard et al., 2007b)

$$
\tilde{\lambda}=\frac{1}{\widetilde{V}_{0}\left(\widetilde{S}_{0}+\widetilde{\alpha} I_{1, \max }\right)^{\tilde{m}}} \int\left(2 T_{0}(\Theta)\right)^{\tilde{m}} d \Theta
$$

where $\widetilde{m}, \widetilde{\alpha}$ and $\widetilde{V}_{0}\left(\widetilde{S}_{0}\right)^{m}$ are three parameters depending on the considered material, and $I_{1, \max }$ the maximum hydrostatic stress over a given cycle.

With a Poisson point process and for a given direction, $\frac{d \widetilde{\lambda}}{d T} V d T$ corresponds to the mean number of sites activated for a shear amplitude ranging from $T$ and $(T+d T)$ in a domain of volume $V$, i.e. the number of sites whose critical shear stress lies between $T$ and $(T+d T)$, and whose dissipated energy during a loading cycle is $\widetilde{\delta}\left(T_{0}, T\right)$. The global dissipated energy density $\tilde{\Delta}$ during a cycle reads

$$
\widetilde{\Delta}=\frac{1}{V} \int_{\Omega} \int_{0}^{T_{a}} \widetilde{\delta}\left(T_{0}, T\right) \frac{d \widetilde{\lambda}}{d T} d T d V=\int_{\Omega} \int_{0}^{T_{a}} \frac{\widetilde{V}_{S}}{\widetilde{h}}\left\langle T_{0}-T\right\rangle T \frac{4 m \int(2 T)^{\widetilde{m}-1} d \Theta}{\widetilde{V}_{0}\left(\widetilde{S}_{0}+\widetilde{\alpha} I_{1, \max }\right)^{\widetilde{m}}} d T d V
$$

As for Equation (10), one introduces a heterogeneity factor to rewrite the previous expression. Equation (21) is then related to $\widetilde{\Sigma}_{0}^{e q}=\underset{n, m}{\operatorname{Max}}\left(2 T_{0}\right)$, Tresca's equivalent stress amplitude, by

$$
\widetilde{\Delta}=\frac{4 \widetilde{V}_{s} \tilde{m}}{\widetilde{h}(\widetilde{m}+1)(\widetilde{m}+2)} \frac{\left(\widetilde{\Sigma}_{0 M}^{e q} \tilde{G}_{\tilde{m}+2}\right)^{\tilde{m}+2}}{\left(\widetilde{V}_{0}^{1 / \widetilde{m}}\left(\widetilde{S}_{0}+\widetilde{\alpha} I_{1, \max M}\right)\right)^{\tilde{m}}}
$$

where

$$
\widetilde{\Sigma}_{0 M}^{e q}=\max _{M \in \Omega}\left[\widetilde{\Sigma}_{0}^{e q}(M)\right] \quad \text { and } \quad I_{1, \max M}=\max _{M \in \Omega}\left[I_{1, \max }(M)\right]
$$

The dissipation heterogeneity factor is now defined by 


$$
\widetilde{G}_{\widetilde{m}+2}=\frac{1}{V} \int_{\Omega}\left(\frac{\widetilde{S}_{0}+\widetilde{\alpha} I_{1, \max M}}{\widetilde{S}_{0}+\widetilde{\alpha} I_{1, \max }(M)}\right)^{m}\left(\frac{\widetilde{\Sigma}_{0}^{e q}(M)}{\widetilde{\Sigma}_{0 M}^{e q}}\right)^{\widetilde{m}+2} \kappa(\widetilde{m}+2) d V,
$$

where $\kappa(\tilde{m}+2)$ represents the distribution of activated directions

$$
\kappa(\widetilde{m}+2)=\int\left(\frac{2 T_{0}(M, \Theta)}{\widetilde{\Sigma}_{0}^{e q}(M)}\right)^{\widetilde{m}+2} d \Theta
$$

It is then possible to rewrite $\tilde{\Delta}$ as

$$
\widetilde{\Delta}=\frac{4 \widetilde{V}_{s} \tilde{m}}{\widetilde{h}(\tilde{m}+1)(\tilde{m}+2)} \frac{\left(\widetilde{\Sigma}_{\text {effiss }}\right)^{\tilde{m}+2}}{\widetilde{V}_{0}\left(\widetilde{S}_{0}\right)^{\tilde{m}}}
$$

where

$$
\widetilde{\Sigma}_{e f f d i s s}=\frac{\widetilde{G}_{\widetilde{m}+2}^{1 /(\widetilde{m}+2)} \widetilde{\Sigma}_{0 M}^{e q}}{\left(1+\frac{\widetilde{\alpha}}{\widetilde{S}_{0}} I_{1, \max M}\right)^{\widetilde{m} /(\widetilde{m}+2)}}
$$

is the "effective dissipation stress." Even though they may appear alike, $\Sigma_{\text {effdiss }}$ and $\widetilde{\Sigma}_{\text {effdiss }}$ are different. The definitions of the equivalent stresses are not the same ( $\Sigma_{0}^{e q}$ is von Mises' equivalent stress, whereas $\widetilde{\Sigma}_{0}^{e q}$ is Tresca's equivalent stress) and $\kappa$ is relevant only for the critical shear stress approach. Consequently the values of the heterogeneity factors $G_{m+2}$ and $\widetilde{G}_{\widetilde{m}+2}$ are also different. Moreover, the parameters $m$ and $\tilde{m}$ are stricto sensu different. However this difference is of no consequence from a practical point of view, i.e. $m$ and $\tilde{m}$ will have the same meaning at the macroscopic scale as one will see hereafter. Beyond these differences of notation, it is worth remembering that the fundamental distinction between the two models lies in the description of microplastic activation.

\section{2-Self-heating under cyclic loading}

The previous sections have described the basis of both models, i.e. from the mechanism of microplasticity activation to the dissipated energy calculation. The two following sections will now present the macroscopic consequences of microplastic activity for each model. First the thermal response ("mean" point of view), and second the fatigue behaviour ("probabilistic" point of view) within the same framework. 
It is assumed that the temperature is uniform in tested samples, which has been theoretically discussed and experimentally checked in the case of tubular specimens in torsion [26]. The mean dissipation $\Delta^{*}$ is then introduced in the following heat conduction equation

$$
\dot{\theta}+\frac{\theta}{\tau_{e q}}=\frac{f_{r} \Delta^{*}}{\rho c}
$$

where $\theta=T_{\text {specimen }}-T_{\text {ref }}$ is the mean temperature variation with respect to the reference temperature $T_{r e f}, \tau_{e q}$ a characteristic time depending on the heat transfer boundary conditions (Chrysochoos and Louche, 2000), $\rho$ the mass density, $c$ the specific heat and $f_{r}$ the loading frequency. There is no need to add a thermoelastic term, which vanishes over one cycle because only mean steady-state temperatures are sought. For both models, the mean (uniform) steady-state temperature $\bar{\theta}$ reads

$$
\bar{\theta}=\eta^{*} \frac{m^{*}}{\left(m^{*}+1\right)\left(m^{*}+2\right)} \frac{\left(\Sigma_{\text {effdiss }}^{*}\right)^{m^{*}+2}}{V_{0}^{*}\left(S_{0}^{*}\right)^{m^{*}}},
$$

with $\eta^{*}=\frac{4 f_{r} \tau_{e q}}{\rho c} \frac{V_{S}^{*}}{h^{*}}$. Equation (29) shows that the thermal behaviour depends only on three parameters and on the effective dissipation stress. This expression is similar to the one for uniform tensile loading thanks to the use of effective stress $\Sigma_{\text {effdiss }}{ }^{*}$.

Even though the meaning of the parameters $m$ and $\tilde{m}$ is initially different in each model (the intensity of the Poisson Point Process depends on different microplasticity descriptions), it may be noted that after the integration over all the slip directions in space, the meaning of $\widetilde{m}$ in the critical shear stress approach is equivalent to the meaning of $m$ in the yield surface one. In the next section, it is proposed to use the same framework to model HCF results.

\section{3-Fatigue limit}

To describe fatigue limits, the weakest link theory is considered. The failure probability is then given by the probability of finding at least one active site. By using Equation (1), the failure probability is related to the loading amplitude by

$$
P_{F}^{*}=1-\exp \left[\int_{\Omega}-\lambda^{*} V d V\right] .
$$




\subsection{1- Yield surface approach}

The failure probability corresponds to the probability of finding at least one active site in a given volume $\Omega$. By using Equation (8), the failure probability is related to the loading amplitude

$$
P_{F}=1-\exp \left[\int_{\Omega}-\frac{V}{V_{0}}\left(\frac{\Sigma_{0}^{e q}(M)}{S_{0}+\alpha I_{1, m}(M)}\right)^{m} d V\right],
$$

which corresponds to Weibull's model (Weibull, 1939, 1951). A second heterogeneity factor $H_{m}$ is introduced to simplify the previous equation. It is defined by

$$
H_{m}=\frac{1}{V} \int_{\Omega}\left(\frac{\left(S_{0}+\alpha I_{1, m M}\right)}{\left(S_{0}+\alpha I_{1, m}(M)\right)} \frac{\sum_{0}^{e q}(M)}{\sum_{0 M}^{e q}}\right)^{m} d V,
$$

and stands for a stress heterogeneity factor, contrary to the previously introduced dissipation heterogeneity factor. Equation (31) becomes

$$
P_{F}=1-\exp \left[-\frac{V_{e f f}}{V_{0}}\left(\frac{\Sigma_{0 M}^{e q}}{S_{0}+\alpha I_{1, m M}}\right)^{m}\right]
$$

where $V_{\text {eff }}=V H_{m}$ denotes the effective volume.

It is worth noting that $H_{m}$ and $G_{m+2}$, even though different in their meanings $\left(H_{m}\right.$ stands for a stress heterogeneity factor whereas $G_{m+2}$ for a dissipation heterogeneity factor) are almost identical in their expressions for proportional loadings. Because of the high value of the exponent $m(m=12$ for the present material, see Section 4.1.1), their values will be very close, even more for loadings with uniform mean hydrostatic stresses. Consequently the combined effects of direction and heterogeneity of loading are nearly the same for fatigue and self-heating results, and thus the interpretation of self-heating results becomes easier. Conversely, for non-proportional loadings, $H_{m}$ and $G_{m+2}$ can be very different as shown below. If two uniform loadings with the same equivalent stress amplitude and the same mean hydrostatic stress are considered, the first one proportional and the second one nonproportional, the stress heterogeneity factors are identical but the dissipation heterogeneity factors can be very different. 
Equation (33) allows for the derivation of the mean fatigue limit $\bar{\Sigma}_{\infty}$ (more precisely, von Mises' equivalent amplitude) and the coefficient of variation $C V$ that are used to describe HCF data

$$
\begin{gathered}
\overline{\left(\Sigma_{0}^{\mathrm{eq}}\right)_{\infty}}=\bar{\Sigma}_{\infty}=\left(S_{0}+\alpha I_{1, m M}\right)\left(\frac{V_{0}}{V_{\text {eff }}}\right)^{(1 / m)} \Gamma\left(1+\frac{1}{m}\right), \\
C V=\frac{\overline{\bar{\Sigma}}_{\infty}}{\bar{\Sigma}_{\infty}}=\frac{\sqrt{\Gamma\left[1+\frac{2}{m}\right]-\Gamma^{2}\left[1+\frac{1}{m}\right]}}{\Gamma\left(1+\frac{1}{m}\right)},
\end{gathered}
$$

where $\overline{\bar{\Sigma}}_{\infty}$ is the corresponding standard deviation, and $\Gamma(x)=\int_{0}^{+\infty}(t)^{x-1} \exp (-t) d t$ Euler's (Gamma) function. The scale and stress heterogeneity effects are taken into account thanks to the effective volume $V_{\text {eff }}$ (i.e., the smaller the effective volume, the higher the stress heterogeneity, the higher the mean fatigue limit). On the contrary, the coefficient of variation is independent of scale or heterogeneity, and only a function of $m$, which is the Weibull modulus (Weibull, 1951).

\subsection{2- Critical shear stress approach}

The failure probability corresponds to the probability of finding at least one active slip direction in the given volume. By using Equation (20), the failure probability is related to the shear stress amplitude

$$
P_{F}=1-\exp \left[-\frac{1}{\widetilde{V}_{0}} \iint\left(\frac{2 T_{a}}{\widetilde{S}_{0}+\widetilde{\alpha} I_{1, \max }}\right)^{\widetilde{m}} d \Theta d V\right]
$$

By using a stress heterogeneity factor $\widetilde{H}_{\widetilde{m}}$, Equation (36) becomes

$$
P_{F}=1-\exp \left[-\frac{\widetilde{V}_{e f f}}{\widetilde{V}_{0}}\left(\frac{\widetilde{\Sigma}_{0 M}^{e q}}{\widetilde{S}_{0}+\widetilde{\alpha} I_{1, \max }}\right)^{\widetilde{m}}\right]
$$

which corresponds to Weibull's model (Weibull, 1939). The stress heterogeneity factor $\widetilde{H}_{\widetilde{m}}$ is here defined as 


$$
\widetilde{H}_{\widetilde{m}}=\frac{1}{V} \int_{\Omega}\left(\frac{\left(\widetilde{S}_{0}+\widetilde{\alpha} I_{1, \max M}\right)}{\left(\widetilde{S}_{0}+\widetilde{\alpha} I_{1, \max }(M)\right)} \frac{\widetilde{\Sigma}_{0}^{e q}(M)}{\widetilde{\Sigma}_{0 M}^{e q}}\right)^{\widetilde{m}} \kappa(\widetilde{m}) d V,
$$

so that $\widetilde{G}_{\widetilde{m}+2}$ and $\widetilde{H}_{\widetilde{m}}$ are a priori different because of the influence of the hydrostatic pressure. The values of $\widetilde{G}_{\widetilde{m}+2}$ and $\widetilde{H}_{\widetilde{m}}$ are again nearly identical because of the high value of $\widetilde{m}$ for proportional and non-proportional loadings.

Last, the mean fatigue limit (i.e., Tresca's equivalent stress amplitude) reads

$$
\overline{\left(\widetilde{\Sigma}_{0}^{e q}\right)_{\infty}}=\widetilde{\bar{\Sigma}}_{\infty}=\left(\widetilde{S}_{0}+\widetilde{\alpha} I_{1, \max M}\right)\left(\frac{\widetilde{V}_{0}}{\widetilde{V}_{\text {eff }}}\right)^{(1 / \widetilde{m})} \Gamma\left(1+\frac{1}{\widetilde{m}}\right),
$$

and the coefficient of variation $C V$ is the same as for the previous model (Equation (35)).

Because of the same expression (29) used for the identification of $m^{*}$ (see Section 3.2), the values of $m$ and $\tilde{m}$ are identical. Consequently the fatigue scatter will be the same for both models (same $C V$ value). On the contrary, the mean fatigue limits will be different for the two approaches because Equations (34) and (39) account for the influence of several parameters on fatigue properties (namely, the stress heterogeneity, the volume effect, the hydrostatic stress and the multiaxiality of the loading with the equivalent stress amplitude and the parameter $\kappa(\widetilde{m})$ (Morel, 1998)) in different ways.

Both models depend on three parameters to predict the fatigue limits, and a last one to account for self-heating under cyclic loadings. In the next section, it is proposed to analyse the ability of both models to describe multiaxial self-heating responses in the case of tubular specimens under tension-torsion cyclic loadings, and to evaluate the capacity of both approaches to predict multiaxial fatigue properties.

\section{3-Application to tension-torsion loadings}

Tubular specimens being used, the macroscopic stress tensor depends only on the radius $r$ of the specimen and is defined by

$$
\Sigma(r)=\left(\begin{array}{ccc}
\Sigma_{11,0} \sin \left(2 \pi f_{r} t\right)+\Sigma_{m} & \Sigma_{12,0} \frac{r}{R_{e}} \sin \left(2 \pi f_{r} t+\varphi\right) & 0 \\
\sum_{12,0} \frac{r}{R_{e}} \sin \left(2 \pi f_{r} t+\varphi\right) & 0 & 0 \\
0 & 0 & 0
\end{array}\right)_{\left(\vec{z}, \vec{e}_{\theta},-\vec{e}_{r}\right)},
$$


where $\Sigma_{11,0}$ and $\sqrt{3} \Sigma_{12,0}=\tan (\phi) \Sigma_{11,0}$ are the tensile and shear stress amplitudes, $\varphi$ the phase lag between shear and normal stresses, $\Sigma_{m}$ the mean tensile stress, and $R_{e}$ the external radius of the specimen.

In the particular case of tension-torsion loading on tubular specimens, the hydrostatic pressure is uniform over the volume of the specimen, so that the two heterogeneity factors of the first model have the same expression for proportional loadings

$$
G_{m}=H_{m},
$$

and the two heterogeneity factors of the second model are identical for proportional and nonproportional loadings

$$
\widetilde{G}_{\widetilde{m}}=\widetilde{H}_{\widetilde{m}}
$$

\section{1 - Experimental results}

The tested material is a medium carbon steel C45 (SAE45) whose chemical composition is given in Table 1, and monotonic tensile properties in Table 2. The microstructure is made of equi-axed grains with no significant texture. All multiaxial self-heating tests are performed on the same type of specimens, taken from the same bar of steel in a region whose grain size and hardness uniformity was checked. The elastic and thermal parameters are considered uniform and isotropic. The specimens are tubular, with a thickness / mean radius of the gauge section equal to 0.24 (Figure 3a). The external surface is ground for all specimens.

All experiments are performed using an MTS tension-torsion servohydraulic-testing machine with a load capacity $100 \mathrm{kN}$ in tension and $1200 \mathrm{Nm}$ in torsion. All loading paths were applied at a frequency of $5 \mathrm{~Hz}$. For temperature measurements and data processing, the multiaxial self-heating procedure is similar to earlier uniaxial experiments (Doudard et al., 2005). One uses two (K type) thermocouples to measure the temperatures of the gauge zone and the lower grip (Figure 3b), and a ThermoEst TTE 50P/A conditioner.

Table 1: Chemical composition of C45 (SAE45) steel $\left(10^{-2} \mathrm{wt} \%\right)$.

\begin{tabular}{cccccccc}
\hline $\mathrm{C}$ & $\mathrm{Mn}$ & $\mathrm{Si}$ & $\mathrm{Cr}$ & $\mathrm{P}$ & $\mathrm{S}$ & $\mathrm{Mo}$ & $\mathrm{Fe}$ \\
\hline $45-51$ & $50-80$ & $15-35$ & $<40$ & $<3$ & $2-4$ & $<10$ & balance \\
\hline
\end{tabular}


Table 2: Mechanical properties of C45 (SAE45) steel. $Y_{s}=$ yield stress;

$U T S=$ ultimate tensile strength; $e l=$ elongation at failure.

\begin{tabular}{ccc}
\hline$Y_{S}(\mathrm{MPa})$ & UTS $(\mathrm{MPa})$ & el $(\%)$ \\
\hline$>360$ & $>700$ & $28 \%$ \\
\hline
\end{tabular}
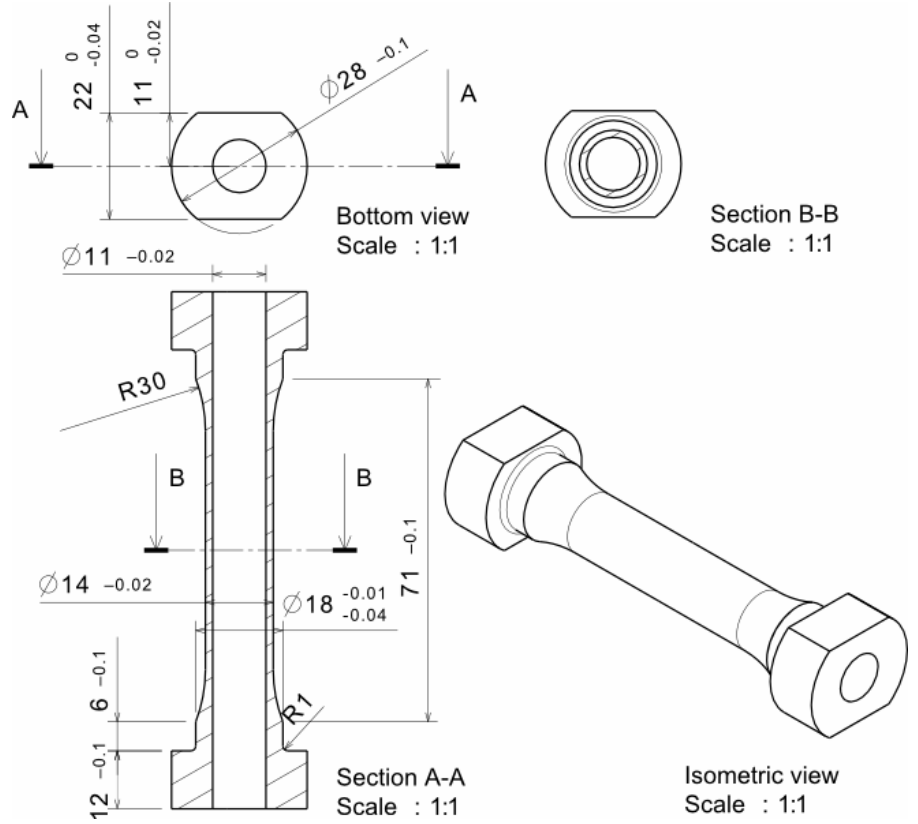

$\mathbf{- a -}$

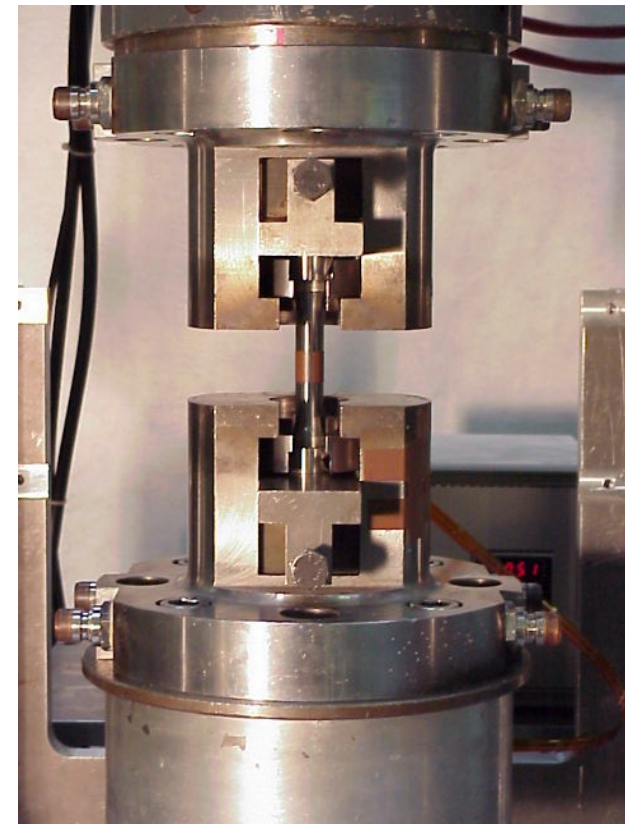

$-b-$

Figure 3: Tubular specimen geometry in mm (a). Experimental set-up: specimen, grips, thermocouples, conditioner (b).

Tests begin when the grip temperature is stabilised (i.e., after the testing machine has warmed up for several hours). During the test, the amplitude of loading is step-wise constant, and increases once the differential temperature is stabilised ( $c a .3000$ cycles in the present case). More detailed descriptions of the experimental set-up are given in Refs. (Poncelet, 2007; Poncelet et al., 2007).

Four different loading paths are performed. Pure tension and pure torsion are first applied, both with zero mean stress, then pure tension with non-zero mean stress, and an outof phase tension-torsion loading with a constant von Mises' equivalent stress at mean radius $\left(\phi=48^{\circ}, \varphi=90^{\circ}\right)$. The steady-state temperature is plotted as a function of the loading amplitude in Figure 4. 


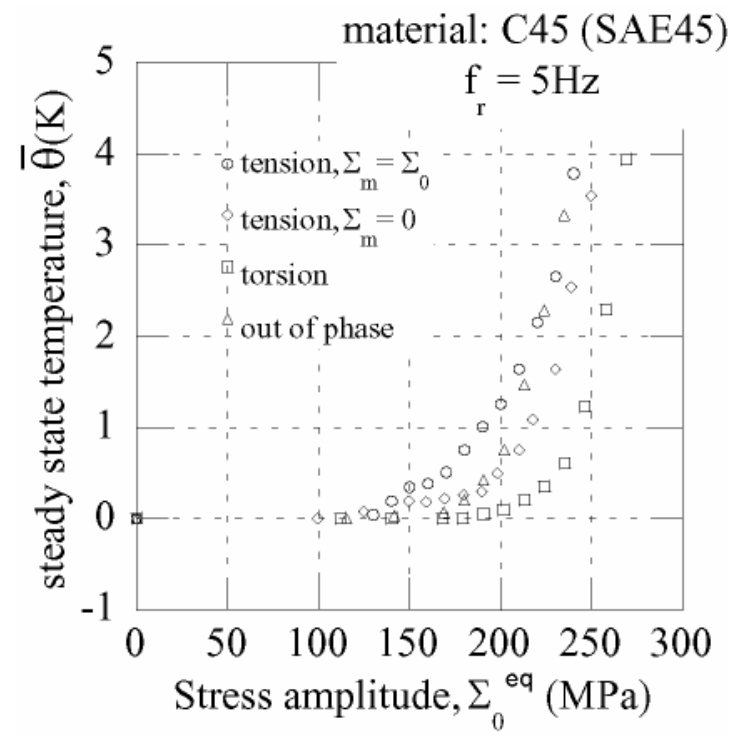

Figure 4: Steady state temperatures as functions of the equivalent stress amplitude for different loading paths.

As for other steels (Doudard et al., 2005; Doudard, 2004; Poncelet, 2007), each curve has a first part that shows virtually no change in temperature, whereas in the second part the temperature increases significantly with the stress amplitude. This transition is reported to be a rather good estimation of the mean fatigue limit for steels in uniaxial homogeneous case (Doudard et al., 2004; Krapez et al., 1999; La Rosa and Risitano, 2000; Luong, 1995). Moreover it has been shown that the gradual increase of temperature is linked to the fatigue scatter (Doudard et al., 2004). The relatively short duration of self-heating tests (in comparison with traditional fatigue tests) makes them not only interesting for academic studies, but also very attractive for industrial purposes.

It is now proposed to check whether the links between thermal response and fatigue properties are still found in multiaxial cases, and more precisely if an adequate model identified on thermal responses is able to predict fatigue limits.

\section{2-Identification procedure}

Both models depend exactly on the same parameters, namely, $m$ or $\widetilde{m}$ describe the scatter of fatigue results and the slope (in a log-log plot) of the self-heating temperature response, $\eta$ or $\tilde{\eta}$ are scale parameters for the thermal response, $\alpha$ or $\widetilde{\alpha}$ account for the effect of the mean hydrostatic stress on self-heating and fatigue properties, and $V_{0}\left(S_{0}\right)^{m}$ or $\widetilde{V}_{0}\left(\widetilde{S}_{0}\right)^{\tilde{m}}$ are scale parameters for the fatigue response. All parameters will be identified using only 2 selfheating tests and one fatigue limit that may come from any type of geometry or loading (it is 
nonetheless possible to perform identification only with fatigue results if self-heating ones are not available).

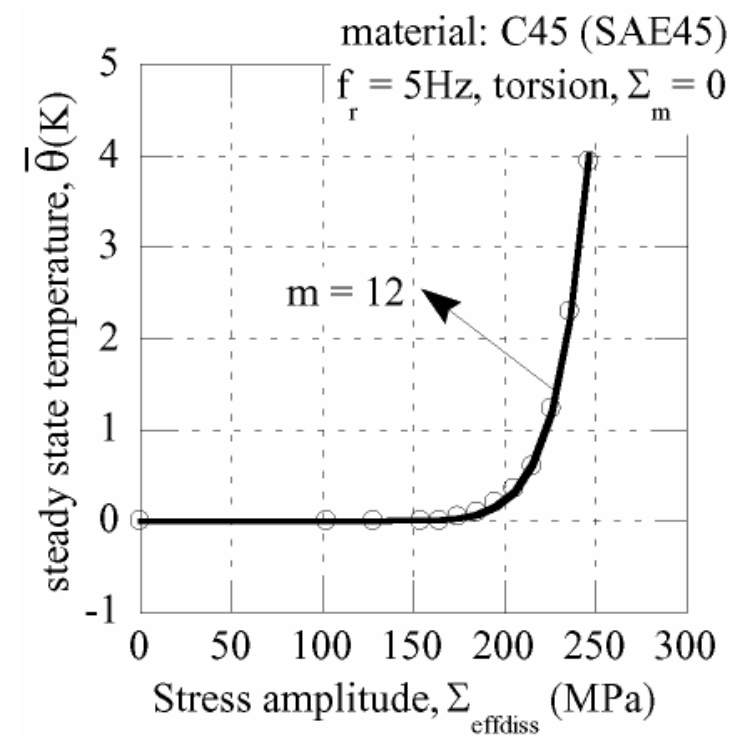

$-a-$

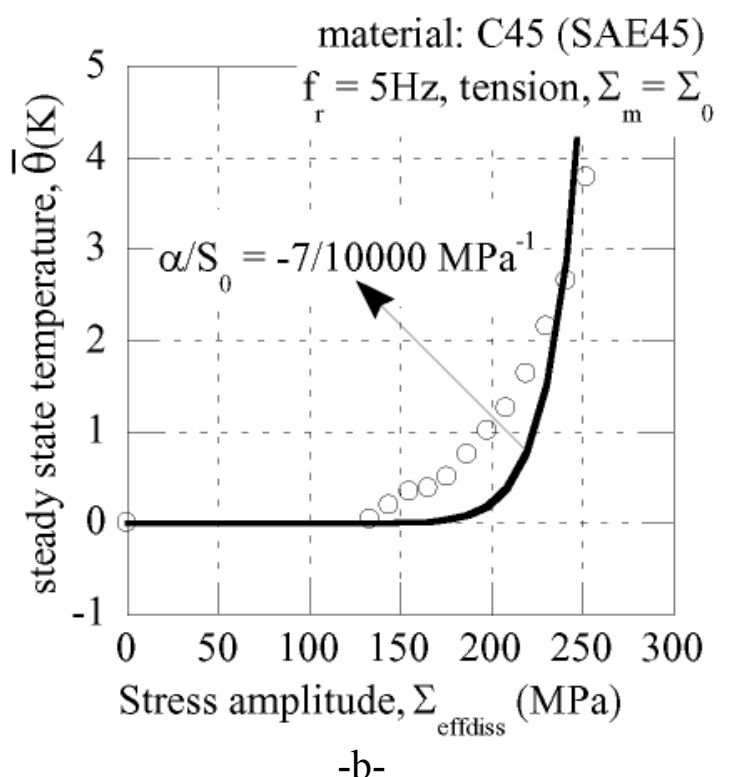

-b-

Figure 5: Identification of the parameters $m, \eta$ and $\alpha$ (yield surface approach) from the self-heating test for two loading paths: torsion (a) and tension (b).

Figure 5a shows the identification of the parameters $m$ and $\eta$ of the yield surface based model using a pure torsion self-heating curve $\left(\phi=90^{\circ}\right.$ and $\left.\Sigma_{m}=0\right)$ and Equation (29). The calculation of $H_{m+2}$ is then possible, and the parameter $\alpha$ is determined from a different selfheating test result, in the present case (Figure 5b) pure tension $\left(\phi=0^{\circ}\right.$ and $\left.\Sigma_{m}=\Sigma_{0}\right)$. Following the same procedure (a pure torsion self-heating test $\left(\phi=90^{\circ}\right.$ and $\left.\Sigma_{m}=0\right)$ and pure tension $\left(\phi=0^{\circ}\right.$ and $\left.\left.\Sigma_{m}=\Sigma_{0}\right)\right)$, parameters $\tilde{m}, \tilde{\eta}$ and $\tilde{\alpha}$ of the microscopic model are identified (Figure 6). 


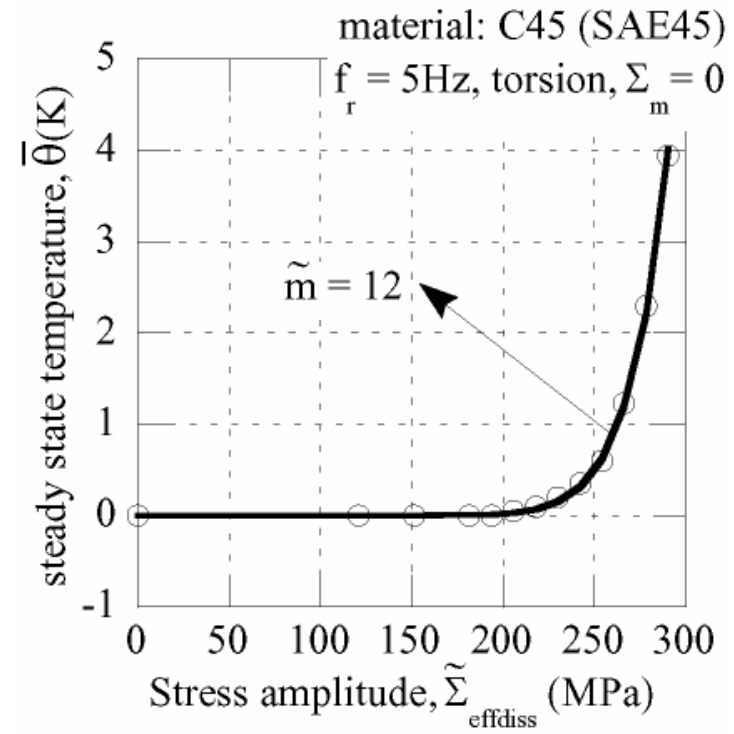

$-\mathrm{a}-$

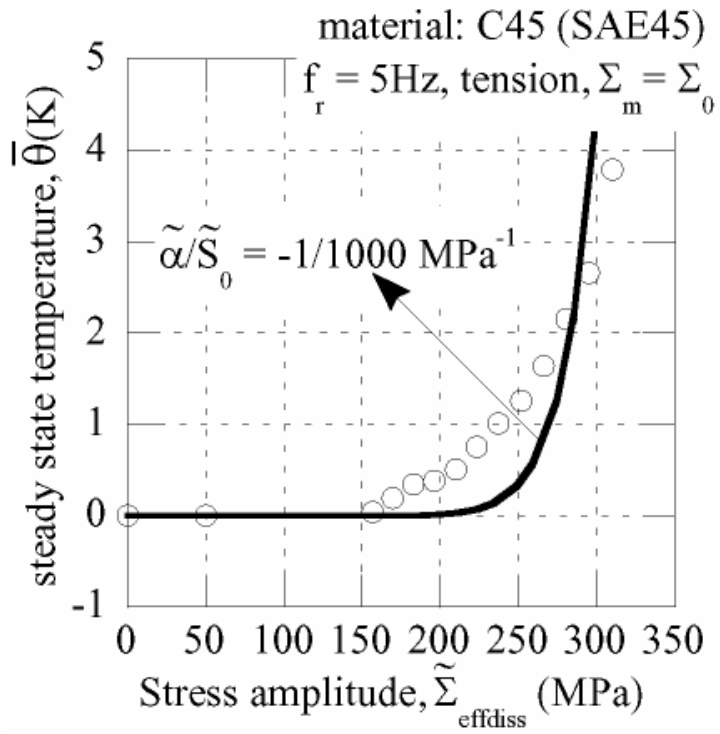

$-b-$

Figure 6: Identification of the parameters $\tilde{m}, \widetilde{\eta}$ and $\widetilde{\alpha}$ (critical shear stress approach) from the self-heating tests for two loading paths: torsion (a) and tension (b).

It is worth noting that no non-proportional loading results are needed to tune the material parameters. More generally, any couple of loading paths may be used to identify the yield surface (resp. critical shear stress) based model parameters $m, \eta$ and $\alpha$ (resp. $\tilde{m}, \tilde{\eta}$ and $\widetilde{\alpha}$ ), as long as their mean (resp. maximum) hydrostatic stresses over a given cycle are different.

The last (scale) parameter of each model is obtained by using a mean fatigue limit and Equation (34) for the yield surface approach, or Equation (39) for the critical shear stress approach. This value is obtained for tensile loadings $\left(\phi=0^{\circ}\right.$ and $\left.\Sigma_{m}=0\right)$ when fatigue limits are evaluated for $5 \times 10^{6}$ cycles. Staircase tests are performed on 15 smooth and round samples machined from the same steel bar as before on a $50 \mathrm{kN}$-servohydraulic testing machine with a loading frequency ranging from $20 \mathrm{~Hz}$ to $60 \mathrm{~Hz}$. Stress steps are equal to $10 \mathrm{MPa}$. The measured mean fatigue limit is $262 \mathrm{MPa}$.

\section{3-Predictions of multiaxial self-heating}

First, one checks the prediction of self-heating responses under proportional loadings. An "iso-temperature surface" description is proposed (Doudard et al., 2007a). It consists in a series of proportional self-heating tests applied to the same specimen. The amplitude of loading increases step by step. When the differential temperature reaches a given offset, a new path starts with a different loading direction. The offset is chosen to ensure a stability of the surface and to minimise history effects (Poncelet, 2007). The directions are investigated 
with $\pi / 8$-rad steps in the $\left(\Sigma_{11} ; \sqrt{3} \Sigma_{12}\right)$ space. The first, the $6^{\text {th }}$ and the last loading directions are the same $(0 \mathrm{rad})$ to check that no history effects occur. The offset being reached with a difference of loading amplitude of $2 \%$, one considers the history effect negligible. For each loading path the stress amplitude for which the differential temperature reaches the given offset is depicted by a line in the $\left(\Sigma_{11} ; \Sigma_{12}\right)$ space (Figure 7$)$. The measured iso-temperature surface shown in Figure 7 was obtained for an offset of $0.5 \mathrm{~K}$. The $2^{\text {nd }}, 3^{\text {rd }}$ and $4^{\text {th }}$ directions (respectively equal to $\pi / 8,2 \pi / 8$ and $3 \pi / 8 \mathrm{rad}$ ) are expected to give the same results as the $7^{\text {th }}$, $8^{\text {th }}$ and $9^{\text {th }}$ directions (the surface is nearly symmetric with respect to the shear axis). Figure 8 shows the comparisons of both models against the experimental results. The difference between model and experiment is less than $2.9 \%$ (resp. $2.1 \%$ ) for the yield surface approach (resp. critical shear stress approach), which proves the validity of their predictions when proportional loadings are considered.

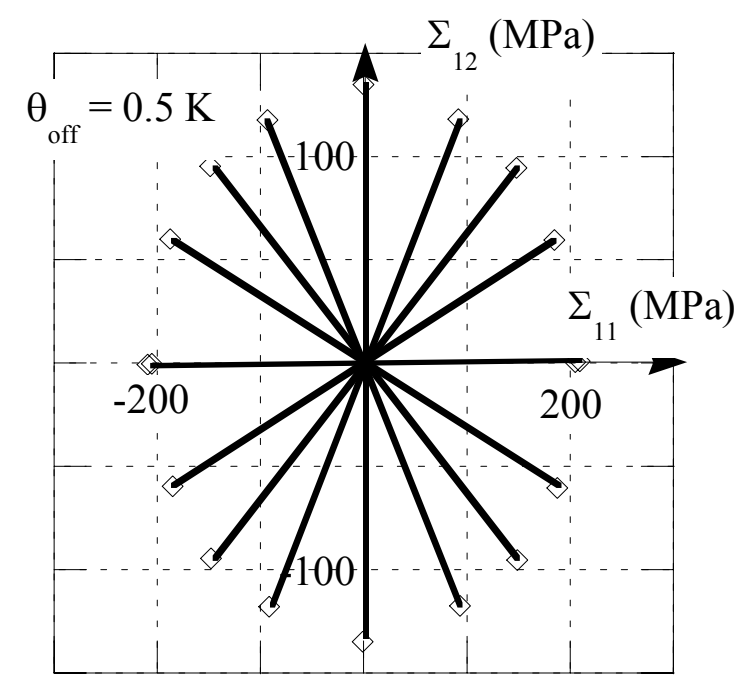

Figure 7: Measured iso-temperature surface for an offset of $0.5 \mathrm{~K}$.

Second, the prediction of the complete self-heating response under proportional and non-proportional loadings is investigated. When the effective dissipative stress of each model is used to plot the four self heating results (Figure 9), one observes that the different selfheating curves collapse onto one another. In particular, the out-of-phase loading that produced a significantly higher temperature compared with the other loading paths lies on top of the other responses. This result proves that the non-proportionality of the loading is well accounted for by the yield surface and critical shear stress approaches. It is worth noting that these good predictions were obtained with both approaches. The yield surface approach 
predicts the same number of active sites for two uniform loadings with the same equivalent stress amplitude and the same hydrostatic stress, the first one proportional and the second one non-proportional. Nonetheless the dissipation heterogeneity factors are different so that the experimental self-heating results are well predicted. For the critical shear stress approach, the numbers of active sites are different because of the presence of the $\kappa$ factor.

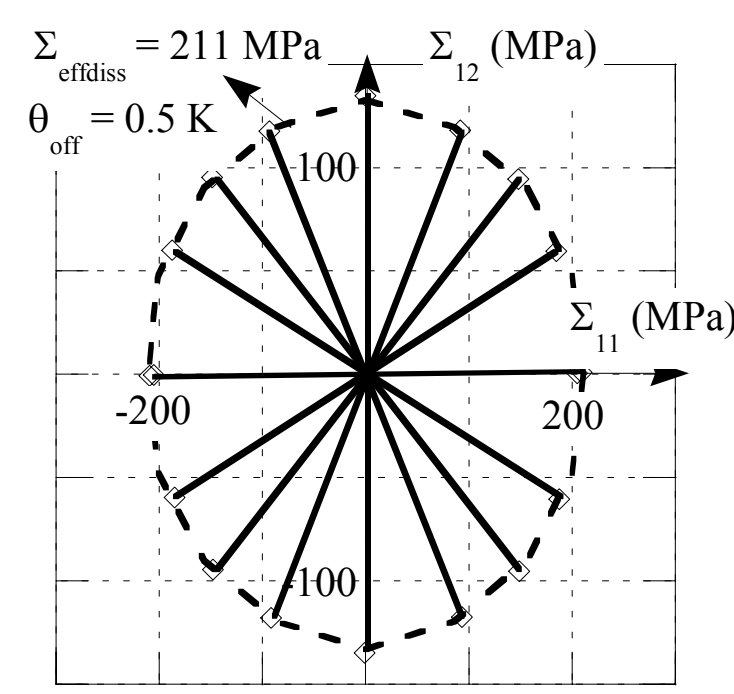

-a-

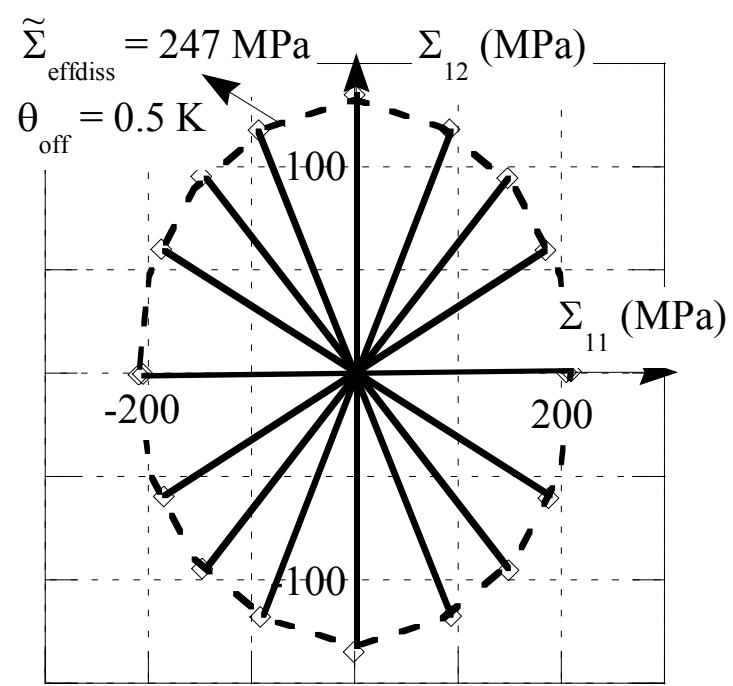

$-b-$

Figure 8: Comparison of yield surface based model (a) surface and critical shear stress based model (b) surface with measured one.

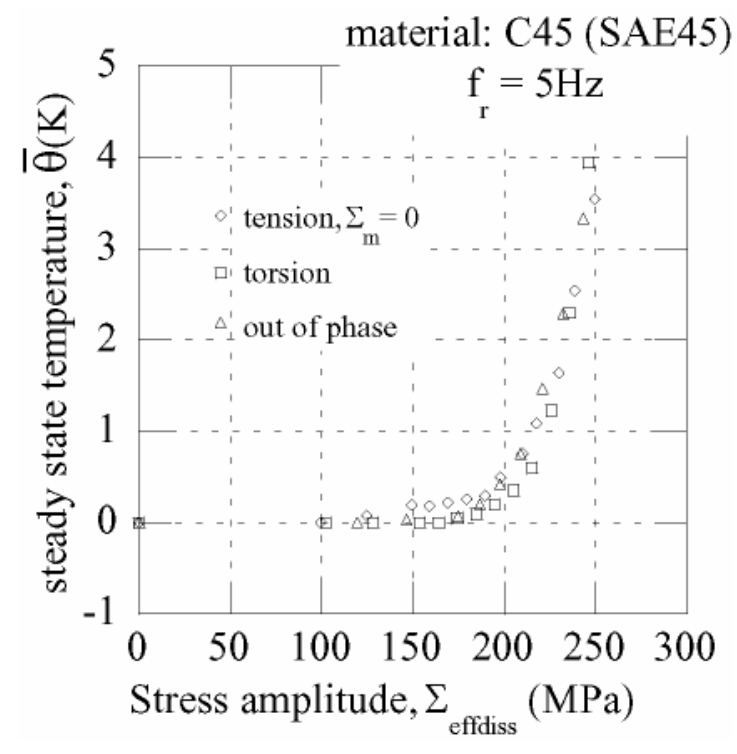

-a-

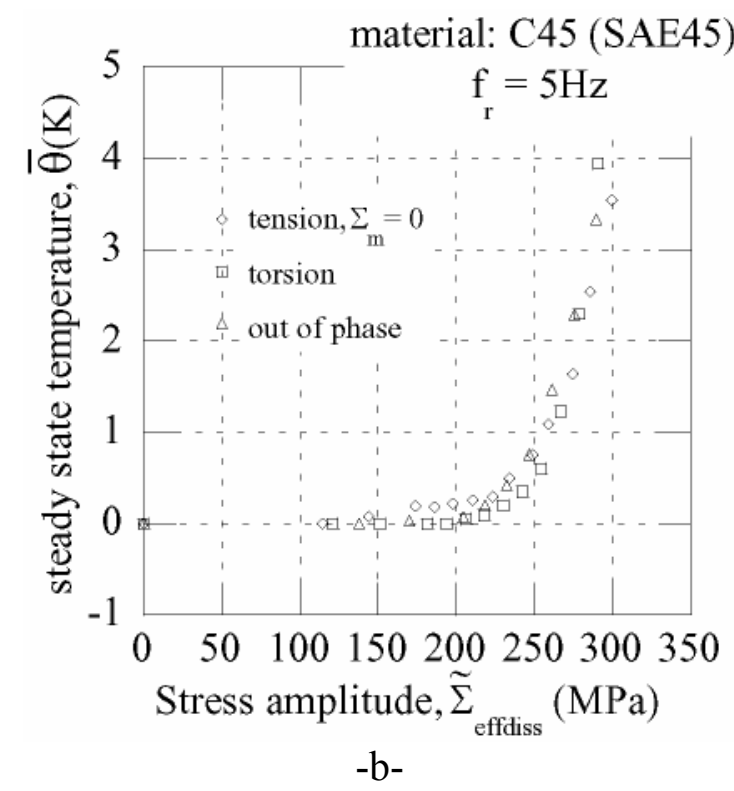

$-b-$

Figure 9: Steady state temperatures as functions of the effective dissipative stress for the yield surface (a) and critical shear stress (b) approaches. 


\section{4-Predictions of multiaxial fatigue limits}

To check the multiaxial fatigue limits predicted by the models, three series of staircase tests (target: $5 \times 10^{6}$ cycles) are performed. The first one uses pure torsion loading. The second one corresponds to $\phi=48^{\circ}$ and $\Sigma_{m}=0$, i.e. a proportional tension-torsion test. The last one is a non-proportional loading corresponding to $\phi=48^{\circ}, \varphi=90^{\circ}$ (i.e., an out-of-phase loading path where the points at mean radius of the specimen are loaded with a constant von Mises' equivalent stress). All the tests are carried out on the same testing machine as for the selfheating tests, at a loading frequency of $20 \mathrm{~Hz}$. For the staircase tests, the fatigue limits are calculated with the Dixon-Mood method (Lieurade, 1982) (Table 3). The corresponding fatigue limits are then predicted by both models for each loading path. Table 3 shows the relative prediction error $(R P E)$ defined by

$$
\mathrm{RPE}=\frac{\bar{\Sigma}_{\infty}^{\exp }-\bar{\Sigma}_{\infty}^{\mathrm{pre}}}{\bar{\Sigma}_{\infty}^{\exp }}
$$

for both models.

Table 3: Relative prediction errors of both models.

\begin{tabular}{cccc}
\hline Loadings $\left(\Sigma_{\mathrm{m}}=0\right)$ & $\begin{array}{c}\text { Prop. } \\
\left(\phi=90^{\circ}\right)\end{array}$ & $\begin{array}{c}\text { Prop. } \\
\left(\phi=48^{\circ}\right)\end{array}$ & $\begin{array}{c}\text { Non-prop. } \\
\left(\phi=48^{\circ}, \varphi=90^{\circ}\right)\end{array}$ \\
\hline Mean fatigue limits, $\Sigma_{0}^{\mathrm{eq}}(\mathrm{MPa})$ & 277 & 267 & 205 \\
\hline Critical shear stress approach & $6 \%$ & $7 \%$ & $-5 \%$ \\
\hline Yield surface approach & $9 \%$ & $6 \%$ & $-17 \%$ \\
\hline
\end{tabular}

\section{5-Discussion}

Both thermal and fatigue behaviours are well described by each model. Relative errors are small for each experimental validation, even though the "identification cost" is low (and the same for each model), thanks to the information coming from the self-heating tests.

Although fundamentally different in their microplasticity description, the distinction between the yield surface and critical shear stress approaches is nearly impossible from a thermal point of view (see Figures 6 and 7). When the fatigue behaviour is studied, a distinction is possible. On the one hand, errors of multiaxial fatigue limit predictions are less than $17 \%$ for the yield surface approach. On the other hand, the critical shear stress approach 
yields errors less than 7\%. Concerning proportional loading paths, the two models are equivalent. For the non-proportional loading, the predictions are non-conservative in both cases. However the interest of the critical shear stress approach is clearly shown, lowering (in absolute value) the prediction error from $-17 \%$ to $-5 \%$ without the addition of new parameters. When considering the tensile loading path as a reference for the first model, Table 4 shows that the dissipation heterogeneity factors are more sensitive to nonproportional loading paths than the stress heterogeneity factors so that this model leads to good predictions of self-heating but not so good for fatigue limits. For the critical shear stress approach, the heterogeneity factors are alike (the influence of non-proportionality is taken into account in the heterogeneity factors by the $\kappa$ parameter). Figure 10 shows the sensitivity of the $\kappa$ parameter to non-proportional loadings. These results confirm that even if the global structure of the model is left unchanged, the description of microplasticity at the scale of the slip-planes by using a probabilistic approach is the key ingredient to better capture fatigue limits under non-proportional loadings.

Table 4: Values of the different heterogeneity factors when $m=12$ for the studied specimen.

\begin{tabular}{|c|c|c|c|c|c|}
\hline \multicolumn{2}{|c|}{$\begin{array}{c}\text { Loadings Factors } \\
(\mathrm{m}=12)\end{array}$} & \multicolumn{3}{c|}{ Proportional $\left(\varphi=0^{\circ}\right)$} & Non-Proportional \\
\cline { 4 - 7 } & $\phi=0^{\circ}$ & $\phi=48^{\circ}$ & $\phi=90^{\circ}$ & $\phi=48^{\circ}, \varphi=90^{\circ}$ \\
\hline $\begin{array}{c}\text { Stress } \\
\text { (Yield surf. appr.) }\end{array}$ & $H_{m}$ & 1 & 0.55 & 0.36 & 0.42 \\
\hline $\begin{array}{c}\text { Dissip. } \\
\text { (Yield surf. appr.) }\end{array}$ & $G_{m+2}$ & 1 & 0.5 & 0.32 & 1.48 \\
\hline $\begin{array}{c}\text { Stress } \\
\text { (Critical shear appr.) }\end{array}$ & $\frac{\widetilde{H}_{\widetilde{m}}}{\widetilde{H}_{\widetilde{m}}\left(\phi=90^{\circ}, \varphi=0\right)}$ & 8 & 2.3 & 1 & 2.6 \\
\hline $\begin{array}{c}\text { Dissip. } \\
\text { (Critical shear appr.) }\end{array}$ & $\frac{\widetilde{G}_{\widetilde{m}+2}}{\widetilde{G}_{\widetilde{m}+2}\left(\phi=90^{\circ}, \varphi=0\right)}$ & 10.2 & 2.5 & 1 & 2.65 \\
\hline
\end{tabular}




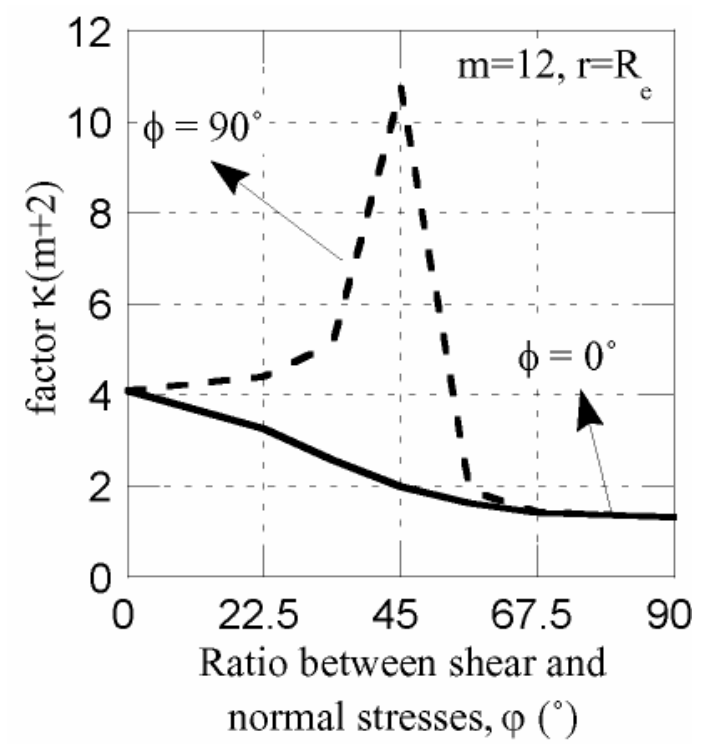

Figure 10: Factor $\kappa$ as a function of the ratio between shear and normal stresses $\phi$ for a proportional loading $\left(\varphi=0^{\circ}\right)$ and an out-of-phase $\left(\varphi=90^{\circ}\right)$ path.

\section{4-Conclusion}

This work is part of a general study on the links between self-heating responses and high cycle fatigue properties. The present paper was devoted to the comparison of two multiscale models whose parameters are tuned thanks to self-heating data, and subsequently used to predict multiaxial high cycle fatigue properties. The first model uses an equivalent stress to account for stress multiaxiality at microscopic scale, whereas the second one relies on a description of microplasticity at the scale of slip-planes.

Both models are identified using two different self-heating tests and one fatigue limit. Any type of loading can be used for these tests as long as the maximum (for the critical shear stress approach) or mean (for yield surface approach) hydrostatic stress over a cycle is different between the two self-heating tests. The identification procedure is thus "low cost" and easily applicable to already existing results, provided the (dissipative) mechanisms at play in self-heating and fatigue tests are identical.

The models relate thermal effects induced by microplastic dissipation to fatigue properties under multiaxial cyclic loadings. They account for the influence of the stress heterogeneity, the volume effect and the hydrostatic stress on fatigue properties. The validation of the models is obtained in terms of mean fatigue limits and temperature histories for in and out-of phase tension-torsion loadings. In the case of non-proportional loadings, the critical shear stress approach leads to good predictions of fatigue limits whereas the yield surface approach is less reliable. This ability is not due to the slip plane approach itself (it 
would lead to an equivalent model if a deterministic point-of-view were chosen), but to the combination of slip-plane plasticity and probabilistic activation.

The next step of this work is the prediction of multiaxial fatigue life with the critical shear stress approach and the application to industrial cases.

\section{Acknowledgment}

The authors would like to thank ArcelorMittal Global R\&D and Nippon Steel Corporation for their technical and financial support. 


\section{References}

Bainvillet, A., Palin-Luc, T., Lasserre, S., 2003. A volumetric energy based high cycle multiaxial fatigue criterion. Int. J. Fat. 25, 755-769.

Bérard, J.-Y., Rathery, S., Béranger, A.-S., 1998. Détermination de la limite d'endurance des matériaux par thermographie infrarouge. Mat. Techn. 1-2, 55-57.

Berveiller, M., Zaoui, A., 1979. An extension of the selfconsistent scheme to plastically flowing polycristals. J. Mech. Phys. Solids. 26, 325-344.

Cazaud, R., 1948. La fatigue des métaux. ed. Dunod, Paris (France).

Chrysochoos, A., Louche, H., 2000. An infrared image processing to analyse the calorific effects accompanying strain localisation, Int. J. Engrg. Sci. 38, 1759-1788.

Cugy, P., Galtier, A., 2002. Microplasticity and temperature increase in low carbon steel, in: Proceedings of the 7th Int. Fat. Conf., Stockholm (Sweden), 2002.

Cura, F., Curti, G., Sesana, R., 2005. A new iteration method for the thermographic determination of fatigue limit in steels. Int. J. Fat. 27 [4], 453-459.

Curtin W.A., 1991. Exact Theory of Fiber Fragmentation in Single-Filament Composite, $J$. Mater. Sci., 26, 5239-5253.

Dang Van, K., 1973. Sur la résistance à la fatigue des métaux. Sciences et techniques de l'armement, Mémorial de l'artillerie française, 3ème fascicule.

Dengel, D., Harig, H., 1980. Estimation of the fatigue limit by progressively-increasing load tests. Fat. Fract. Eng. Mat. Struct. 3 [1], 113.

Denoual, C., Hild, F., 2002. Dynamic fragmentation of brittle solids: A multi-scale model. Eur. J. Mech. A/Solids. 21, 105-120.

Doudard, C., 2004. Détermination rapide des propriétés en fatigue à grand nombre de cycles à partir d'essais d'échauffement. PhD thesis, ENS Cachan, France.

Doudard, C., Calloch, S., Hild, F., Cugy, P., Galtier, A., 2004. Identification of the scatter in high cycle fatigue from temperature measurements. C.R. Mécanique. 332 [10], 795801.

Doudard, C., Calloch, S., Cugy, P., Galtier, A., Hild, F., 2005. A probabilistic two-scale model for high cycle fatigue life predictions. Fat. Fract. Eng. Mat. Struct. 28, 279288.

Doudard, C., Poncelet, M., Calloch, S., Boué, C., Hild, F., Galtier, A., 2007a. Determination of an HCF criterion by thermal measurements under biaxial cyclic loading, Int. J. Fatigue. 29 (4), 748-757. 
Doudard, C., Hild, F., Calloch, S., 2007b. A probabilistic model for multiaxial high cycle fatigue. Fat. Fract. Eng. Mat. Struct. 30, 107-114.

Eshelby, J.D., 1957. The Determination of the Elastic Field of an Ellipsoidal Inclusion and Related Problems. Proc. Roy. Soc. London A. 241, 376-396.

Fedelich B., 1998. A stochastic theory for the problem of multiple surface crack coalescence, Int. J. Fract., 91, 23-45.

Galtier, A., Bouaziz, O., Lambert, A., 2002. Influence de la microstructure des aciers sur leurs propriétés mécaniques. Méc. Ind. 3 [5], 457-462.

Gulino, R., Phoenix, S.L., 1991. Weibull strength statistics for graphite fibres measured from the break progression in a model graphite/glass/epoxy microcomposite. J. Mater. Sci. 26, 3107-3118.

Harry, R., Joubert, F., Gomaa, A., 1981. Measuring the Actual Endurance Limit of One Specimen Using a Nondestructive Method. J. Eng. Mat. Tech. 103 [1], 71-76.

Jeulin, D., 1991. Modèles morphologiques de structures aléatoires et changement d'échelle. thèse d'Etat, Université de Caen, France.

Kanarchuk, V.E., Dmitriev, N.N., Derkachev, O.B., Zhelnov, G.N., 1989. Thermal radiation of steel specimens under cyclic loading. Strength of materials. 21 [2], 202-207.

Krapez, J.-C., Pacou, D., Bertin, C., 1999. Application of lock-in thermography to a rapid evaluation of the fatigue limit in metals. 5th AITA, Int. Workshop on Advanced Infrared Techn. and Appl., Venezia (Italy), ed. E. Grinzato et al., 379-385.

Kröner, E., 1984. On the plastic deformation of polycristals. Acta Met. 9, 155-161.

La Rosa G., Risitano, A., 2000. Thermographic methodology for rapid determination of the fatigue limit of materials and mechanical components. Int. J. Fat. 22 [1], 65-73.

Lehr, E., 1926. Die Dauerfestigkeit, ihre Bedeutung für die Praxis und ihre Kurzfristige Ermittlung mittels neuartiger Prüfmaschinen. Glasers Ann. Gew.1184, 109-114.

Lemaitre, J., Doghri, I., 1994. Damage 90: a post processor for crack initiation. Comput. Methods Appl. Mech. Engrg. 115, 197-232.

Lieurade, H.P., 1982. La pratique des essais de fatigue. first ed. PYC, Paris (France).

Luong, M.P., 1995. Infrared thermographic scanning of fatigue in metals. Nuclear Engineering and Design. 158 [2-3], 363-376.

Mabru, C., Chrysochoos, A., 2001. Dissipation et couplages accompagnant la fatigue de matériaux métalliques. Photomécanique 2001, ed. Y. Berthaud, M. Cottron, J.-C. Dupré, F. Morestin, J.-J. Orteu and V. Valle, GAMAC, 375-382. 
Moore, H.F., Kommers, J.B., 1921. Fatigue of metals under repeated stress. Chem. Met. Eng. $25,1141-1144$.

Morel, F., 1998. A fatigue life prediction method based on a mesoscopic approach in constant amplitude multiaxial loading. Fat. Fract. Eng. Mat. Struct. 21, 241-256.

Poncelet, M., 2007. Multiaxialité, hétérogénéités intrinsèques et structurales des essais d'autoéchauffement et de fatigue à grand nombre de cycles. PhD thesis, ENS Cachan, France.

Poncelet, M., Doudard, C., Calloch, S., Hild, F., Weber, B., Galtier, A., 2007 Prediction of self-heating measurements under proportional and non-proportional multiaxial cyclic loadings, C. R. Mécanique. 335, 81-86.

Sobczyk K., Kirkner D.J., 2001. Stochastic Modeling of Microstructures, Birkhäuser, Boston (USA).

Stärk, K.F., 1980. Thermometrische Untersuchungen zum zyklischen Verformungsverhalten metallischer Werkstoffe. Ph.D. Thesis, Universität Stuttgart, Germany.

Stromeyer, C.E., 1915. A machine for determining fatigue limits calorimetrically. Rep. Brit. Ass. 638.

Weibull, W., 1939. A Statistical Theory of the Strength of Materials, Roy. Swed. Inst. Eng. Res., Report 151, 1939.

Weibull, W., 1951. A statistical distribution function of wide applicability, ASME J. Appl. Mech. 18, 293-297.

Welter, G., 1937. Essais d'endurance par traction et compression. Wlad. Inst. Met. 4, 30-39.

Yang, B., Liaw, P.K., Morrison, M., Liu, C.T., Buchanan, R.A., Huang, J.Y., Kuo, R.C., Huang, J.G., Fielden, D.E., 2005. Temperature evolution during fatigue damage. Intermetallics. 13, 419-428. 